\title{
A Bioinformatics Analysis of Gene Expression Changes in Human Alzheimer's Disease and Mouse Models
}

\author{
Kaishi $\mathrm{Xu}^{1}$, Yingyue $\mathrm{Zhou}^{2+}$ \\ ${ }^{1}$ Suzhou Foreign Language School, kevinxu233@outlook.com \\ ${ }^{2}$ Washington University in St. Louis, yingyue@wustl.edu \\ ${ }^{\dagger}$ Corresponding author: Yingyue Zhou
}

\begin{abstract}
Alzheimer's disease (AD), the most common form of dementia, affects more than 50 million people worldwide, with no current treatment to halt the disease. The exact molecular mechanisms modulating disease progression remains elusive, even though numerous studies using mouse AD models have been done. In addition, as mouse models do not fully recapitulate human pathology, it is unclear to what extent results acquired from mouse models can be applied to treat humans. In this study, we conducted comprehensive bioinformatics analyses on transcriptomic profiles from mice bearing Abeta or tau pathology and human AD to identify differentially expressed genes (DEGs) and biological pathways shared among them. We identified the disease-associated microglia (DAM) signature and inflammatory pathways in both amyloid and tau mouse models compared to controls. Although GFAP was the only DEG shared by human AD and mouse $\mathrm{AD}$ models, pathways such as inflammatory response were identified in both human and mouse. Common pathways found in this study may modulate disease progression and provide new therapeutic targets.
\end{abstract}

\section{CCS CONCEPTS}

- Applied computing Life and medical sciences $\sim$ Bioinformatics - Applied computing Life and medical sciences $\sim$ Genomics $\sim$ Computational genomics

Permission to make digital or hard copies of all or part of this work for personal or classroom use is granted without fee provided that copies are not made or distributed for profit or commercial advantage and that copies bear this notice and the full citation on the first page. Copyrights for components of this work owned by others than ACM must be honored. Abstracting with credit is permitted. To copy otherwise, or republish, to post on servers or to redistribute to lists, requires prior specific permission and/or a fee. Request permissions from Permissions@acm.org. BIC 2021, January 22-24, 2021, Harbin, China

(C) 2021 Association for Computing Machinery. ACM ISBN 978-1-4503-9000-2/21/01 ...\$15.00 https://doi.org/10.1145/3448748.3448755

\section{Keywords}

Bioinformatics analysis, Alzheimer's disease, gene expression

\section{INTRODUCTION}

Alzheimer's disease (AD) is a common neurodegenerative disease that has become the sixth leading cause of death in the United States [1]. Sporadic AD, in most cases, occurs after the age of 65 , and patients suffer from memory loss, cognitive function decline and even difficulty swallowing and walking at later stages. Molecularly, the aggregation of Abeta polypeptides in the brain is hypothesized as the main cause of $\mathrm{AD}$, which drives formation of hyper-phosphorylated tau tangles [2]. Both amyloid plaques and fibrillary tangles are evident in AD patient brains.

Abeta polypeptides were first found in 1984 as primary constituents of meningovascular amyloid [2]. These peptides are cleaved by beta- and gamma- secretases from amyloid precursor protein (APP), and secreted into the extracellular space. Abeta polypeptides are prone to aggregate into sheet conformations in the form of higher-order oligomers, protofibrils, and fibrils, which are detectable in AD brains. Accumulation of tau tangles follows Ab aggregation. Tau is a microtubule associated protein. Hyperphosphorylation of tau results in perturbation of microtubule functions and aggregation of neurofibrillary tangles, both of which contribute to neurodegeneration. Although aging is the major risk factor for developing $\mathrm{AD}$, genetic components also contribute to it. In familial AD, mutations in either the APP gene or PSEN1, encoding subunits of gamma-secretase lead to increased production of Abeta and thus AD [3].

To study disease progression, multiple AD mouse models have been established that fall in two categories, amyloid pathology and tau pathology [4], to recapitulate the two molecular hallmarks in human pathology. Gene expression profiling studies have been employed in the mouse models to identify factors involved in AD progression [5,6,7]. However, how the various mouse models differ from each other and how well do they mimic pathology in human patients remain questionable. 
In this study, we conducted comprehensive bioinformatics analysis to compare gene expression changes between $\mathrm{AD}$ and control brains in mouse models and human. We identified common genes differentially regulated in both Abeta and tau mouse models. When comparing the mouse transcriptome with human data, although only one gene, GFAP, was commonly upregulated in AD in the datasets we examined, several pathways related to inflammatory response were shared by human and mouse. The shared pathways shed light on molecular mechanisms of AD progression.

\section{RESULTS}

\subsection{Disease progression varies in multiple mouse models of AD}

To determine the disease progression in various mouse models of $\mathrm{AD}$, we focused on the most commonly used models recapitulating Abeta and/or tau pathology (Table 1). Among the models we chose, APPPS1, APPswe, Tg2576 and 5XFAD mice bear the human APP and/or PSEN1 transgenes with AD mutations and develop amyloid pathology, whereas P301S and hTau mice carry human MAPT gene and acquire tau pathology. In addition, the $3 x T g$ mouse model develops both amyloid and tau pathologies, as seen in human AD patients. We compared each of the mouse models from five aspects: plaque deposition, tangle formation, microgliosis, astrogliosis and cognitive impairment.

Table 1. Summary of commonly used AD mouse models.

\begin{tabular}{|c|c|c|c|c|c|c|c|}
\hline $\begin{array}{l}\text { Model } \\
\text { Name }\end{array}$ & Transgene & $\begin{array}{l}\text { Amyloid } \\
\text { pathology }\end{array}$ & Tau & Microgliosis & Astrogliosis & $\begin{array}{l}\text { Neuronal } \\
\text { loss }\end{array}$ & $\begin{array}{l}\text { Behavioral } \\
\text { defects }\end{array}$ \\
\hline APPPS1 & $\begin{array}{c}\text { APP } \\
\text { KM670/671N } \\
\text { L (Swedish) }\end{array}$ & $\begin{array}{c}\text { 6w. cortex } 3- \\
4 \mathrm{~m} . \\
\text { Hippocampus }\end{array}$ & $\begin{array}{c}\text { Phosphorylati } \\
\text { on observed. } \\
\text { No mature }\end{array}$ & $\begin{array}{l}\text { 6w. à Abeta } \\
\text { deposits }\end{array}$ & Increasing & Not observed & $\begin{array}{c}7 \mathrm{w} \text {. à spatial } \\
\text { learning and } \\
\text { memory } 8 \mathrm{w} . \\
\text { Reversal learning } \\
\text { of food rewarded } \\
\text { four arm spatial } \\
\text { maze task }\end{array}$ \\
\hline $5 x F A D$ & $\begin{array}{c}\text { APP } \\
\text { KM670/671N } \\
\text { L (Swedish) }\end{array}$ & $\begin{array}{c}2 \mathrm{~m} \text {. Subiculum } \\
\text { and layer V } \\
\text { cortex }\end{array}$ & absent & $2 \mathrm{~m}$. Gliosis & $2 \mathrm{~m}$. Gliosis & $\begin{array}{c}4 \mathrm{~m} . \\
\text { Presynaptic } \\
\text { marker } \\
\text { synaptophysi } \\
\text { n decline 9m. } \\
\text { Syntaxin, } \\
\text { presynaptic } \\
\text { marker and } \\
\text { PSD-95 } \\
\text { decline }\end{array}$ & $\begin{array}{l}\text { impairment at Y- } \\
\text { maze test }\end{array}$ \\
\hline $\operatorname{Tg} 2576$ & $\begin{array}{c}\text { APP } \\
\text { KM670/671N } \\
\text { L (Swedish) }\end{array}$ & $\begin{array}{c}11-13 \mathrm{w} . \\
\text { Parenchymal } \\
\text { plagues }\end{array}$ & absent & $\begin{array}{c}10-16 \mathrm{~m}, \\
\text { hippocampus, } \\
\text { frontal cortex, } \\
\text { entorhinal } \\
\text { cortex, occipital } \\
\text { cortex }\end{array}$ & & absent & $\begin{array}{c}<6 \mathrm{~m} \text {. Spatial } \\
\text { learning working } \\
\text { memory } \\
\text { contextual fear } \\
\text { conditioning }\end{array}$ \\
\hline APPswe & $\begin{array}{c}\text { APP } \\
\text { KM670/671N } \\
\text { L (Swedish) }\end{array}$ & $\begin{array}{l}\text { 6w. Deposit 9w. } \\
\text { Hippocampus } \\
12 \mathrm{w} \text {. Increasing }\end{array}$ & absent & & $\begin{array}{l}3 \mathrm{~m} \text {. Few } 6 \mathrm{w} . \\
\text { Significant } 15 \mathrm{w} \\
\text { Cortex }\end{array}$ & $\begin{array}{l}\text { near plague } \\
\text { with distal } \\
\text { area }\end{array}$ & $\begin{array}{l}\text { impairment at } \\
\text { morris water maze }\end{array}$ \\
\hline
\end{tabular}




\begin{tabular}{|c|c|c|c|c|c|c|c|}
\hline $\begin{array}{l}\text { Model } \\
\text { Name }\end{array}$ & Transgene & $\begin{array}{l}\text { Amyloid } \\
\text { pathology }\end{array}$ & Tau & Microgliosis & Astrogliosis & $\begin{array}{l}\text { Neuronal } \\
\text { loss }\end{array}$ & $\begin{array}{l}\text { Behavioral } \\
\text { defects }\end{array}$ \\
\hline htau & $\begin{array}{l}\text { MAPT, Mapt } \\
\text { knock out }\end{array}$ & absent & $\begin{array}{c}9 \mathrm{~m} . \mathrm{Tau} \\
\text { tangle } 6 \mathrm{~m} . \\
\text { Hyperphosph } \\
\text { orylated tau }\end{array}$ & unknown & unknown & $\begin{array}{l}\text { 10-14w. } \\
\text { Decrease in } \\
\text { cortical } \\
\text { thickness }\end{array}$ & $\begin{array}{l}6 \mathrm{~m} . \text { Abnormal } \\
\text { spatial learning }\end{array}$ \\
\hline P301S & MAPT P301S & absent & $\begin{array}{c}6 \mathrm{~m}, \\
\text { neocortex, } \\
\text { amygdala, } \\
\text { hippocampus, } \\
\text { brain stem } \\
\text { and spinal } \\
\text { cord }\end{array}$ & $\begin{array}{c}3 \mathrm{~m} \text {, white } \\
\text { matter of brain } \\
\text { and spinal cord. } \\
6 \mathrm{~m} \text {. Increase in } \\
\text { white and gray } \\
\text { matter of } \\
\text { hippocampus, } \\
\text { amygdala, } \\
\text { entorhinal } \\
\text { cortex, and } \\
\text { spinal cord }\end{array}$ & $\begin{array}{l}\text { following } \\
\text { microgliosis }\end{array}$ & $\begin{array}{l}\text { 3-6m. In CA3 } \\
\text { region of the } \\
\text { hippocampus }\end{array}$ & $\begin{array}{l}\text { impairment at } \\
\text { morris water maze }\end{array}$ \\
\hline $3 \times \mathrm{Tg}$ & $\begin{array}{c}\text { APP } \\
\text { KM670/671N } \\
\text { L (Swedish) }\end{array}$ & $\begin{array}{c}6 \mathrm{~m} . \text { Frontal } \\
\text { cortex }\end{array}$ & $\begin{array}{c}12 \mathrm{~m} . \\
\text { Hippocampus } \\
6 \mathrm{~m} \text {. Absent }\end{array}$ & $\begin{array}{l}7 \mathrm{~m} \text {. Increasing } \\
\text { density }\end{array}$ & unknown & unknown & $\begin{array}{l}\text { impairment at } \\
\text { long-term } \\
\text { retention }\end{array}$ \\
\hline
\end{tabular}

First, from amyloid pathology perspective, APPPS1 and APPswe begin to show Abeta deposits in the cortex at 6 weeks of age, which then spread to the hippocampus at 9 weeks in APPswe mouse model, and at 3-4 months in APPPS1. Compared to APPPS1 and APPswe, Abeta deposits appear later in the cortex in 3xTg at 6 months. Parenchymal plaques are evident in $\operatorname{Tg} 2576$ between 11 to 13 months. Among all amyloid models, 5XFAD is rather aggressive in that extracellular plaque deposition is detected as early as 2 months of age.

Second, from Tau tangling perspective, $\mathrm{Tg} 2576$ and APPswe do not show any tangling present in the brain. P301S mice develop neurofibrillary tangles at 6 months, when htau mice show hyperphosphorylation of tau, and neurofibrillary tangles mature after 9 months in htau miceage. 3xTg mice present Tau tangling in the hippocampus after 12 months.

Third, from gliosis aspects, microgliosis and astrogliosis occur following deposition of plaques or tangles Microgliosis begins to show near Abeta deposition in APPPS1 and 5XFAD at 6 weeks and 2 months, respectively and astrogliosis is present. In Tg2576, microgliosis happens during 10 to 16 months throughout brain regions, such as hippocampus, frontal cortex, entorhinal cortex and also occipital cortex. Astrogliosis is present concurrently in the cortex. In 3xTg mouse model, microgliosis happens after 7 months. Microgliosis occurs at 3 months in P301S and is followed by astrogliosis.
Finally, from behavioral deficits angle, all of the mouse models mentioned above develop cognitive impairments in spatial learning and memory, although the exact onset of the defects is not clear as results differ in different studies.

\subsection{Gene expression profiles change as disease progresses}

To compare gene expression signatures in different mouse models, we chose two representative models, one Abeta model 5xFAD and one Tau model P301S. We first compared among the different disease stages within each model and then compared the similarities between the two models.

To study transcriptional changes along tau progression, we examined gene expression profiles of P301S mice at 3 months, 6 months, 9 months and 12 months of age from GSE 89979 dataset, and identified differentially expressed genes (DEGs) between P301S mice and the control at each stage. 17 genes were differentially expressed at 3 months. The number of DEGs increased to 34 at 6 months, which reached the peak of 227 at 9 months. Then it dropped down to 110 DEGs at 12 months (Figure 1a). We found that many of the DEGs were consistent throughout disease progression, which is shown in the Venn diagram (Figure 1a). 18 DEGs overlapped between 6and 9 - months, the number of which increased to 50 between 9- and 12- months. The DEGs shared by 9- and 12-month-old mice are listed in Figure 1a. 


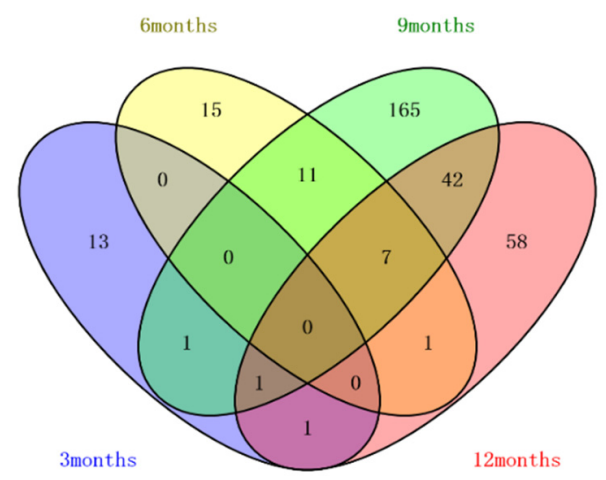

Venn diagram showing shared DEGs from P301S mice at different ages (GSE 89979). 50 overlapped genes between 9- and 12-month are listed at the bottom.

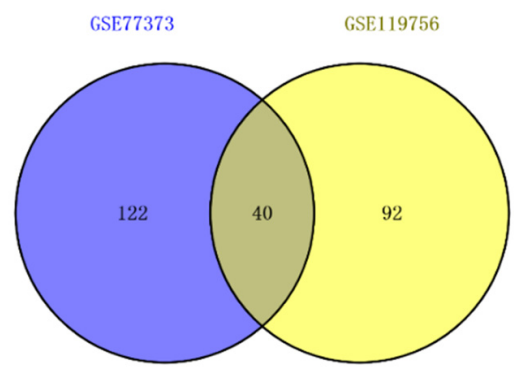

b. Venn diagram showing shared DEGs from datasets GSE 77373 and GSE 119756 of the 5XFAD model. 40 overlapped genes between the two datasets are listed at the bottom.

Figure 1. DEGs are shared among P301S and 5XFAD mouse models.

We went on to study DEGs in amyloid deposition models, between the 5XFAD and control mice. We chose two datasets, GSE77373 and GSE 119756 on the 5XFAD model, to identify DEGs consistent among various datasets. In a total of 162 DEGs from GSE 77373 and 132 DEGs from GSE 119756, 40 of them were shared between the datasets, and are listed in Figure $1 \mathrm{~b}$.

In order to investigate common DEGs between $\mathrm{Ab}$ and tau models, we extracted the shared genes from the fore mentioned models, and found that 12 genes were differentially expressed in both models: Tyrobp, C1qb, Trem2, C1qa, Serpina3n, Mpeg1, Gfap, Osmr, Lyz2, Ccl3, Cd52, and Cst7, all of which were up-regulated in the disease mice compared to control. Majority of the DEGs are highly expressed in microglia (Tyrobp, C1qb, Trem2, C1qa, Mpeg1, Lyz2, Ccl3 Cd52, and Cst7), reflecting inflammation occurring in diseased brains. Furthermore, some DEGs, such as Serpina3n and Gfap, are expressed in astrocytes, which corresponds to astrogliosis observed in mouse models. These results indicate that despite the different pathologies in the two mouse models examined, common transcriptional responses of microglia and astrocytes to $\mathrm{AD}$ are observed.

\subsection{Common pathways are shared by various mouse models}

We then applied gene set enrichment analysis (GSEA) to investigate KEGG and hallmark pathways enriched in each cohort. Top pathways identified based on the upregulated genes in each cohort are listed in Figure 2. Interestingly, all of the top pathways, no matter in which cohort they were enriched, were related to immune functions. For example, inflammatory response, IL2-STAT5 signaling and complement pathways were common in all cohorts. In addition, lysosome pathway was shared between the two datasets on the 5XFAD model. The fact that lysosome pathway was not identified in the tau models indicates that phagolysosomal activity may be more directly associated with Abeta clearance than with tau tangles. Other immune-related pathways, such as cytokinecytokine receptor signaling pathway, were common between Abeta and tau models. 


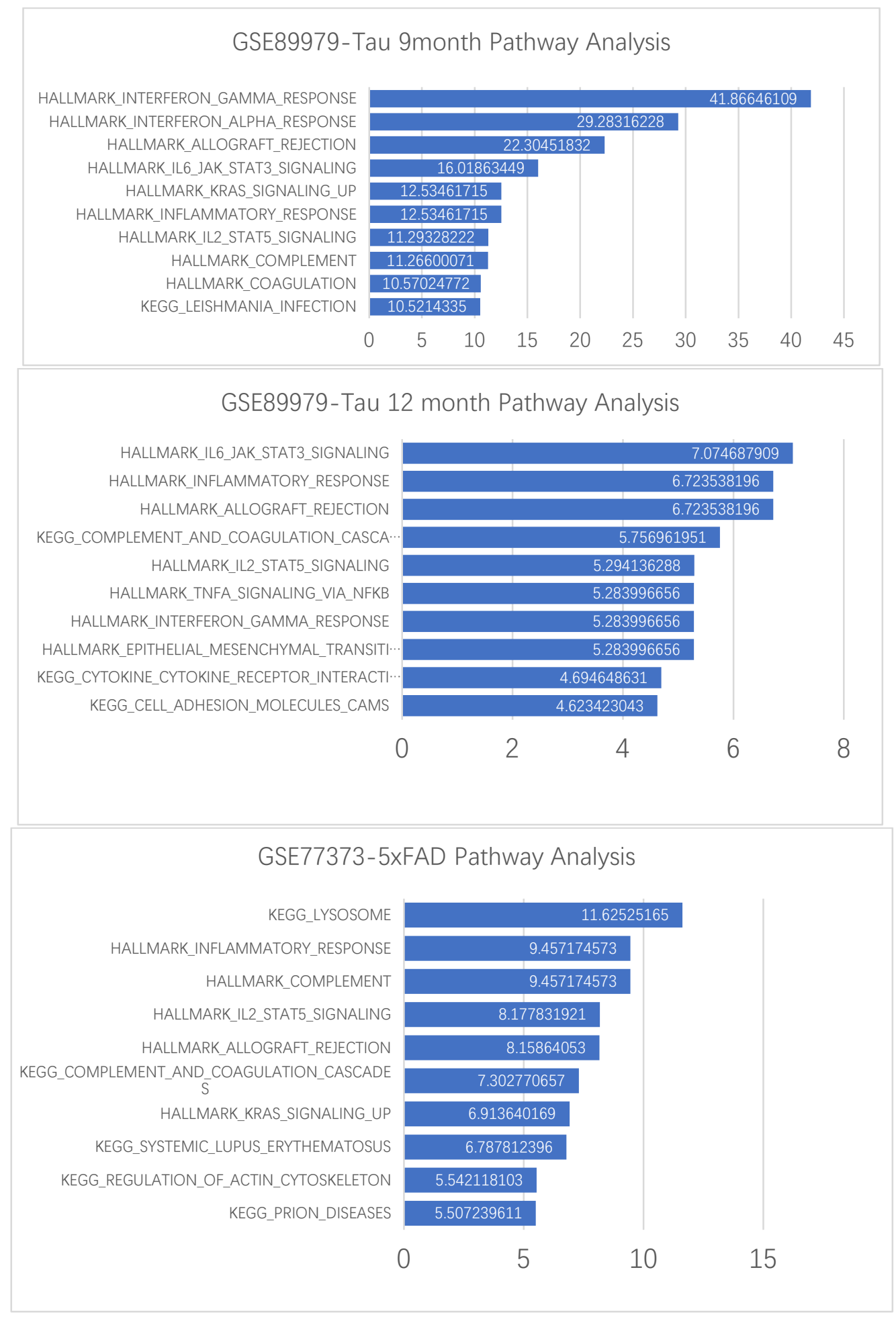




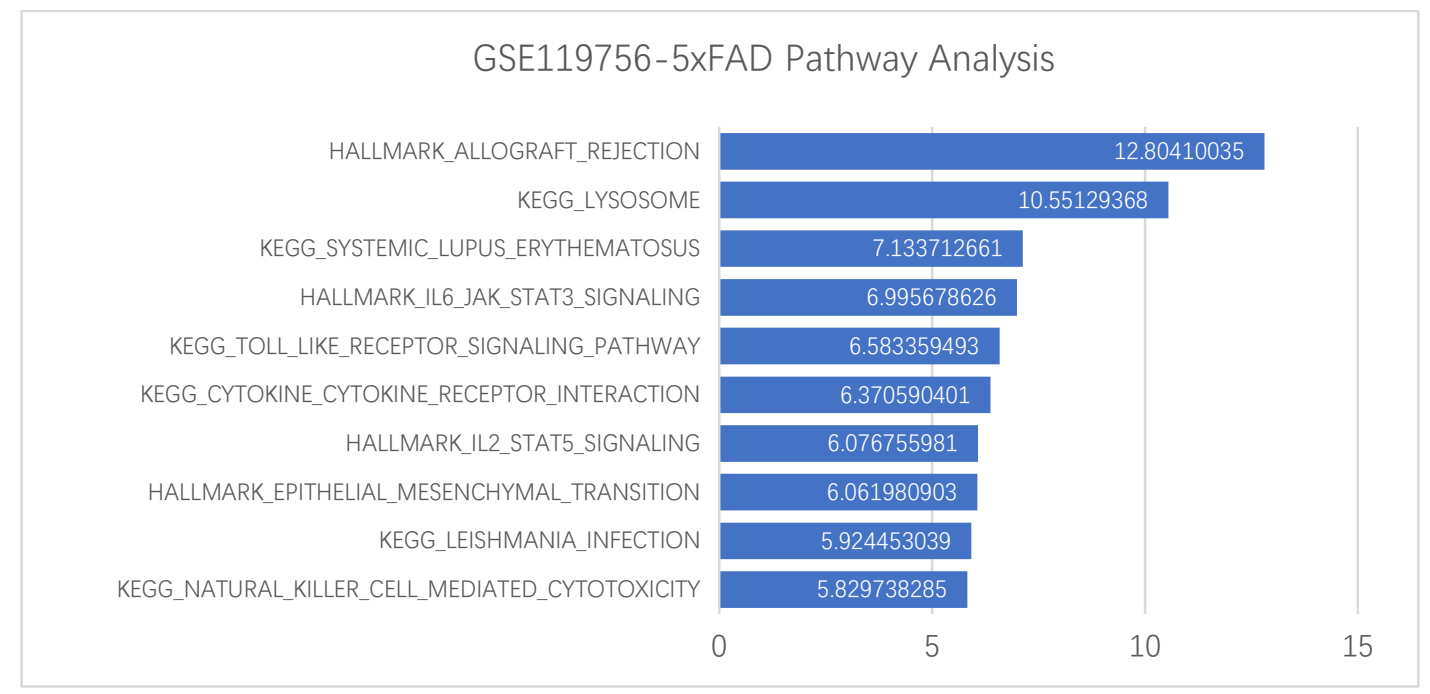

Figure 2. Pathway analysis of top DEGs in the mouse datasets examined. Pathways were calculated by overlap of Hallmark and KEGG gene sets.

\subsection{Common pathways are shared by human AD patients}

To compare our findings in mouse with human data, we examined two databases of human samples that surveyed AD patients and age-matched controls, GSE 118553 and GSE 122063. To account for confounding factors such as source of tissue, we focused on samples from frontal cortex only. In GSE 118553 human dataset, 52 genes were identified as differentially expressed between $\mathrm{AD}$ and control (Figure 3a). GSEA analysis was applied to the DEGs and the top 10 pathways were identified and listed in Figure $3 b$. The top 2 related pathways are glycolysis and epithelial mesenchymal transition. In the other dataset, GSE 122063, 231 DEGs were identified (Figure 3a) and used as inputs for pathway analysis. GSEA analysis indicated complement and inflammatory response as the top pathways upregulated in AD compared to control (Figure 3c). Among the genes differentially expressed between $\mathrm{AD}$ patients and controls, we found that $C D 44, A E B P 1$, $B A G 3, G F A P, H I G D 1 B$ and FCGBP were common in both cohorts of human datasets.

\section{(GSE 122063}

\section{GSE 118553}

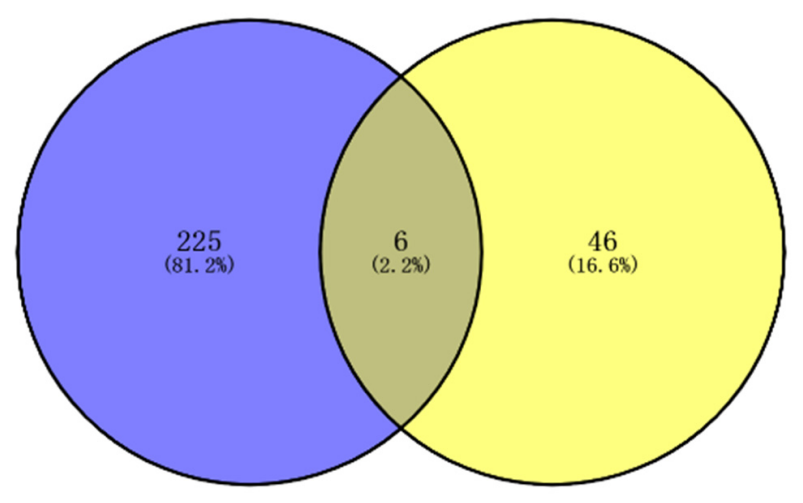

a. Venn diagram showing shared DEGs from datasets GSE 118553 and GSE 122063 of human AD. 


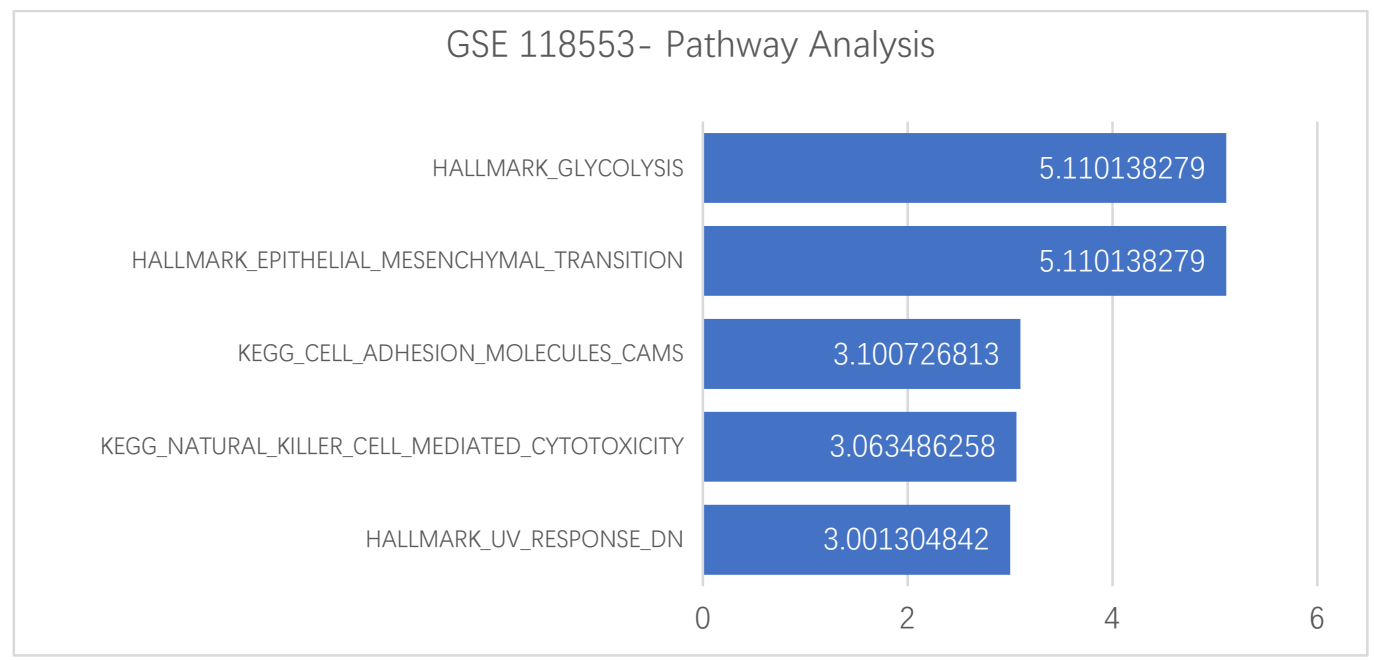

b. Pathways associated with top DEGs in GSE 118553.

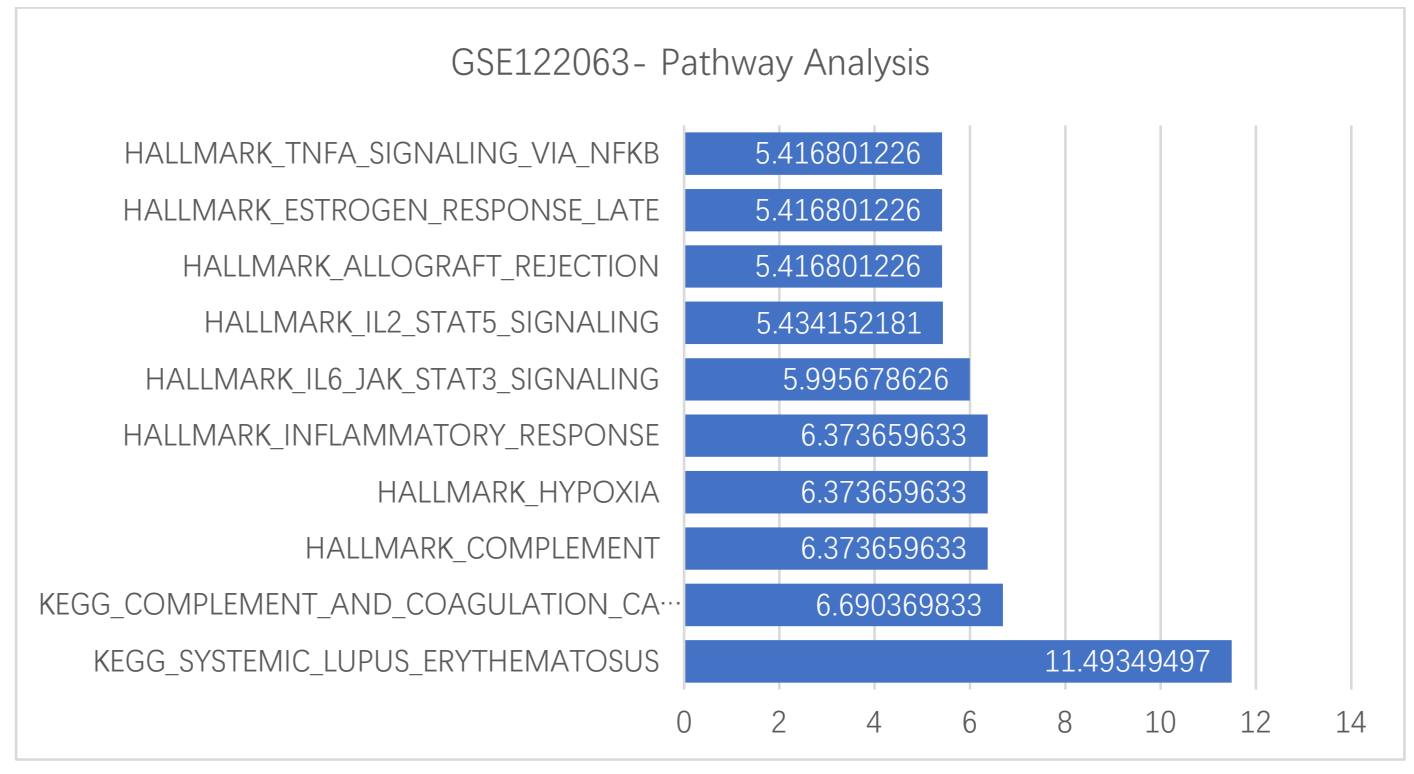

c. Pathways associated with top DEGs in GSE 122063.

Figure 3. DEGs and pathways are shared among various human AD datasets.

\subsection{Similarities between human $A D$ and mouse $A D$ models}

In comparison between the human $\mathrm{AD}$ and mouse $\mathrm{AD}$ models, we set the goal to find similarities between them. We found that GFAP was the only DEG shared by all datasets we examined. Despite the few genes commonly dysregulated in human and mouse, several pathways were similarly enriched in human $\mathrm{AD}$ and mouse AD models, including complement pathway, inflammatory response and IL2-STAT5 signaling pathway.

\section{DISCUSSION}

Various mouse models have been widely used to study molecular mechanisms of AD progression. However, majority of the established models only recapitulate part of the AD pathology, either amyloid or tau, leaving the question to what extent can results discovered in mouse models be applied to therapeutics in human asked. In the current study, we examined gene expression changes in 5XFAD, P301S mouse models and human AD patients and identified common pathways shared among all datasets. 
In the P301S tau model, we found more DEGs between the diseased brain and control at 9 months than either at 6 months or 12 months, suggesting a progressive cellular response to disease as tangles accumulate, which then saturates at a later stage. Between the two datasets on the 5XFAD model we examined, only $16 \%$ of the DEGs were shared by them, even though the two datasets analyzed the same mouse model. This could be due to the different time points the tissues were collected or other technical issues during sample preparation that differed in the two datasets. These results emphasized the importance of data repetition by several research groups.

By comparing Abeta model with tau mice, we showed that Tyrobp, C1qb, Trem2, C1qa, Serpina3n, Mpeg1, Gfap, Osmr, Lyz2, Ccl3, Cd52 and Cst7 were consistently upregulated in both models, when compared to controls. Many of these genes, including Tyrobp, C1qb, Trem2, C1qa, Lyz2, Ccl3, Cd52 and Cst7, were classified as the DAM (disease-associated microglia) signature previously characterized in the 5XFAD model [8]. In addition, Serpina3n and Gfap are markers for astrocyte activation. Altogether, these results suggest that microgliosis and astrogliosis are cellular responses to both amyloid pathology and tau pathology. It is speculated that receptors that recognize broad ligands, such as pattern-recognition receptors, are responsible to activate the DAM signature, as it is induced under both pathologies. The patterns recognized by microglia can include specific structures in protein aggregates, or phospholipids released by dead cells.

Analyzing human gene expression profiles has always been challenging. To minimize confounding factors, we limited our analysis on samples from the prefrontal cortex. Despite the variability in human samples, we found six genes upregulated in $\mathrm{AD}$ versus control in two cohorts of samples. GFAP and CD44 are astrocyte activation markers [9]. AEBP1 is a transcription factor expressed in neurons and astrocytes that regulates NF$\mathrm{kB}$ pathway [9]. Upregulation of HIGD1B, expressed in endothelial cells and induced by hypoxia, suggests the involvement of hypoxia in human AD [10]. FCGBP, encoding an IgG-Fc binding protein, may contribute to inflammation. BAG3 was previously indicated involved in clearance of protein aggregates [11]. Although the exact functions of these proteins need further investigation, dysregulation of these genes reflect gliosis, inflammation and vasculature failure characterized clinically in AD human patients, and thus may play a role in the development or modulation of AD.

Interestingly, we found only one DEG, GFAP, shared by human and mouse in the five datasets we examined, suggesting the robustness of upregulation of GFAP in AD. The fact that we identified so few overlapping genes could reflect different disease responses in human and mouse, or could be due to low sensitivity of the gene expression profiling method utilized. On the other hand, we found several dysregulated pathways shared by human and mouse models, including complement pathway, inflammatory response and IL2-STAT5 signaling pathway, suggesting similar cellular responses occur in both human $\mathrm{AD}$ and mouse $\mathrm{AD}$ models, despite that individual genes differ. These results also emphasize the important role of immune functions in AD pathogenesis. We noticed that the dataset GSE 122063 is quite similar to the mouse datasets, indicating that more datasets need to be investigated.

Our results showed that both Abeta and tau pathologies induce a common DAM signature and astrocyte activation in mouse models. Although few DEGs were shared between human and mouse, AD mouse models can recapitulate changes in biological pathways observed in human AD. These results suggest that when developing new therapeutic treatment towards $A D$, targeting specific pathways instead of specific genes may be considered.

\section{METHODS}

\subsection{Datasets}

Datasets analyzed in this study were identified from the Gene Expression Omnibus (GEO) (https://www.ncbi.nlm.nih.gov/gds) repository. We searched for relevant datasets using the following criteria: Alzheimer's disease, expression profiling by array, Abeta or Tau and mus musculus or homo sapiens. The top datasets, GSE 89979 for P301S, GSE 77373 and GSE 119756 for 5XFAD, GSE 122063 and GSE 118553 for human AD were included in this study.

\subsection{Gene expression analysis}

Selected datasets were loaded to the online interactive gene expression analysis platform Phantasus [12]. Duplicated probes were removed by filtering for the maximal median level of expression across all samples using the Collapse tool. Quality control was done by applying k-means clustering to the datasets and outliers were removed.

\subsection{Differential expression analysis}

We used the Limma package embedded in Phantasus to perform differential expression between AD group and non$\mathrm{AD}$ group. DEGs were identified by $\mathrm{p}$-value $<0.05$ and $\log 2$ (fold change) > 1 . We used Venny (https://bioinfogp.cnb.csic.es/tools/venny/) to find the similarities and differences of the datasets.

\subsection{Pathway analysis}

For pathway analysis, we used gene set enrichment analysis (GSEA)/MSigDB platform to compute overlaps between hallmark gene sets and KEGG gene sets of the DEGs identified in each dataset. DEGs that were upregulated in AD vs control from each dataset were used as inputs and the top 10 most significant pathways were presented. 


\section{References}

[1] Facts and Figures. Alzheimer's Disease and Dementia https://www.alz.org/alzheimers-dementia/facts-figures.

[2] Long, J. M. \& Holtzman, D. M. Alzheimer Disease: An Update on Pathobiology and Treatment Strategies. Cell 179, 312-339 (2019).

[3] Sims, R., Hill, M. \& Williams, J. The multiplex model of the genetics of Alzheimer's disease. Nature Neuroscience 23, 311-322 (2020).

[4] Myers, A. \& McGonigle, P. Overview of Transgenic Mouse Models fo Alzheimer's Disease. Current Protocols in Neuroscience 89, e81 (2019).

[5] Mathys, $\mathrm{H}$. et al. Temporal Tracking of Microglia Activation in Neurodegeneration at Single-Cell Resolution. Cell Rep 21, 366-380 (2017).

[6] Habib, N. et al. Disease-associated astrocytes in Alzheimer's disease and aging. Nature Neuroscience 23, 701-706 (2020).

[7] Zhou, Y. et al. Human and mouse single-nucleus transcriptomics reveal TREM2-dependent and TREM2-independent cellular responses in Alzheimer's disease. Nat. Med. 26,131-142 (2020).

[8] Keren-Shaul, H. et al. A Unique Microglia Type Associated with Restricting Development of Alzheimer's Disease. Cell 169, 1276-1290.e17 (2017)

[9] Shijo, M. et al. Association of adipocyte enhancer-binding protein 1 with Alzheimer's disease pathology in human hippocampi. Brain Pathol 28, 5871 (2018).

[10] Peers, C., Pearson, H. A. \& Boyle, J. P. Hypoxia and Alzheimer's disease. Essays Biochem 43, 153-164 (2007).

[11] Lei, Z., Brizzee, C. \& Johnson, G. V. W. BAG3 facilitates the clearance of endogenous tau in primary neurons. Neurobiol Aging 36, 241-248 (2015).

[12] Zenkova, D., Kamenev, V., Sablina, R., Artyomov, M. \& Sergushichev, A phantasus: Visual and interactive gene expression analysis. (Bioconductor version: Release (3.11), 2020). doi: 10.18129/B9.bioc.phantasus. 